\title{
Spatial and Temporal Evolution of Urban Expansion in Oasis Cities: A Case Study of Ganzhou District in Zhangye City
}

\author{
Xuebin Zhang ${ }^{1, \text { a }}$, Jun Luo ${ }^{1,2, b}$ \\ ${ }^{1}$ College of Geography and Environmental Science, Northwest Normal University, China \\ ${ }^{2}$ College of Resources and Environmental Science, Gansu Agricultural University, China \\ azhangxb428@163.com, bjourneylj6@163.com
}

\begin{abstract}
Keywords: urban expansion; spatio-temporal evolution; characteristics; Ganzhou District, Zhangye City
\end{abstract}

Abstract. Urban land use expansion is the main characteristic of traditional urbanization, and which is one of the most significant features of urbanization in China. Taking the typical oasis town of Ganzhou District in Zhangye City as the research case, the paper quantitatively analyzed the process and characteristics of the spatio-temporal evolution of urban expansion in Ganzhou District from 1987 to 2011 with the help of the expansion speed and intensity model and Equal fan analysis method. The results showed that: (1) From 1987 to 2011, urban land expanded rapidly with an increase in an area of $5305.56 \mathrm{hm}^{2}$, the expansion rate was $2.40 \%$, the expansion intensity index was 0.60 , and the main expansion directions were Northwest, Northeast, and East; (2) there are 5 expansion types, respectively are General stable expansion, low speed expansion, medium-speed extension, rapid expansion and high speed expansion, the expansion direction of each type was different; (3) from 1987 to 1995 , cities and towns mainly expanded to the Southeast and Northwest, and the expansion strength of all directions were significantly different. From 1995 to 2000, the cities and towns were expanded to the South, Northeast, Northwest, and East. From 2000 to 2006, the expansion direction mainly to the North, followed by the Southwest and the East. From 2006 to 2011, the urban expansion mainly extended to the Northwest, followed by the Northeast and the West.

\section{Introduction}

Urbanization is a hot issue in geography studies, the performance of urbanization process in space is the process of expansion of construction land[1]. Therefore, urban land expansion is the spatial expression of urbanization, and which is one of the most significant features of urbanization in China[2]. Most of the early urban geography studies of urban spatial evolution were based on socio-economic statistical data, from the perspective of industry, capital, and information, and focused on qualitative and semi-quantitative analysis. Of the study area, most of which are concentrated in the developed regions [3], and there are relatively few studies focused on the underdeveloped regions. In the study of oasis-type cities and towns, most of which are studied from the perspectives of urban systems[4], the possible factors of urban expansion, and urban development patterns[5,6]. There were insufficient quantitative analyses of the overall expansion of urban land use. With the development of geographic information technology, extensive use of quantitative measurement methods, such as using remote sensing data to find the urban development trend,or using composite indicators, such as expansion speed, expansion intensity, fractal dimension, etc. to evaluate land use changes, it is possible to compare and analyze the urban expasion characteristics in different periods, so that the expansion of cities and towns' research towards to be more quantification[7,8]. Based on previous research results, this paper selects Zhangye City, a typical oasis town, as the research case. With the aid of the expansion speed and intensity model and Equal fan analysis method, this paper quantitatively analysis of the process and characteristics of the spatial-temporal evolution of urban expansion in Ganzhou District from 1987 to 2011, to further reveal the spatial evolution mechanism of urban space, so as to provided a reference for the sustainable development of land use in the process of regional development. 


\section{Overview of the study area}

Ganzhou District of Zhangye City, which locates in the middle of Hexi Corridor. The longitude is between $100^{\circ} 04^{\prime} \sim 100^{\circ} 52^{\prime}$, and the latitude is between $38^{\circ} 32^{\prime} \sim 39^{\circ} 24^{\prime}$, which east adjacent to Shandan and Minle county, south to Yugu Autonomous County, west to Linze County, and north to the Alashan Right Banner of Inner Mongolia Autonomous Region. The district administers a national-level economic and technological development zone, 18 townships, 5 sub-district offices, and 245 administrative villages. The area is $65 \mathrm{~km}$ long from east to west, $98 \mathrm{~km}$ wide from north to south, and has a total area of $3657 \mathrm{~km}^{2}$.

\section{Research Methods}

3.1 Data Sources and Processing The remote sensing data is sourced from Landsat TM 7, provided by the United States USGS website and the Chinese Academy of Sciences Computer Network Information Center geospatial data cloud, which includes the data of 1987, 1995, 2000, 2006 and 2011. Based on the landscape classification system, interpretation is completed through visual aid interpretation. And then we correct the data by using land use maps, Google Earth high-resolution images, and field survey results at different periods in the region, and finally form the land use data of different periods in Ganzhou District. Through the examination of the interpretation results, the overall accuracy is over $90 \%$, which satisfies the precision requirements of the research. The land use classification refers to the Classification of Land Use Status (GB/T21010-2007) and land use/coverage classification system of the national remote sensing monitoring. Combined with the landscape characteristics of the study area, 6 landscape types are included, which are cultivated land, forest land, grassland, construction land, water area and unused land, of which the construction land includes urban land, rural residential land and other construction land.

3.2 Urban expansion model The model includes expansion speed model (M) and expansion intensity model (S), which is used to compare the expansion status of urban land area in different periods, including the expansion strength, speed, and tendency, the formula is:

$$
\begin{aligned}
& M=\frac{A_{i}}{U A \times \Delta t} \times 100 \% \\
& S=\frac{A_{i}}{T A \times \Delta t} \times 100
\end{aligned}
$$

$A_{i}$, which refers to the expansion area of urban land in a certain period; UA is the area of urban land in the initial period; $\Delta t$ is the time span; TA is the total land area of the study area. For the study area is located in the northwestern inland drought region, the proportion of unused land is relatively large, resulting in a small proportion of urban land use in the study area, so the land use change is not significant. In order to better illustrate the expansion of urban land use, the calculation results will be roomed in 10 times.

3.3 Equal fan analysis method With the spatial patterns of cities and towns change in time, there are also some differences showing in the space. We adopt the method of equal-fan analysis and use the city center of Ganzhou District of 1987 as the intersection point, take a certain length as radius (including the scope of the study area), draw four straight lines, and divide the plane space into 8 positions: which are north, northeast, east, southeast, south, southwest, west and northwest, using the extended azimuthal index (OPi) to study the expansion of cities and towns in different directions at different times. The extended azimuth index (OPi) reflects the probability of urban land expansion at a certain position in a certain period of time; the formula is:

$$
O P i=\frac{\left(d_{i} S_{t 2}-d_{i} S_{t 1}\right) \times 100}{S_{\mathrm{t} 2}-S_{t 1}}
$$

OPi is the extended orientation index, which means the proportion of the certain position from $t_{1}$ to $t_{2} ; d_{i} S_{t 1}, d_{i} S_{t 2}$ is respectively the urban land area at time $t_{1}$ and $t_{2} ; S_{t 1}, S_{t_{2}}$ is respectively the total area of the urban land at $\mathrm{t}_{1}$ and $\mathrm{t}_{2}$. 


\section{Results Analysis}

4. 1 Time characteristics of urban expansion Table 1 shows the indexes of urban expansion, from 1987 to 2011, the urban and rural land in Ganzhou District expanded rapidly and the area increased from $9203.20 \mathrm{hm}^{2}$ to $14503.76 \mathrm{hm}^{2}$, increased by $5305.56 \mathrm{hm}^{2}$, the increase proportion was $57.59 \%$, annual average increase of $220.86 \mathrm{hm}^{2}$, the expansion rate was $2.40 \%$, and an expansion intensity index was 0.60 . The proportion of the urban and rural area increased from $2.51 \%$ to $3.96 \%$.

Tab. 1 Urban expansion of Ganzhou County in different periods

\begin{tabular}{lcccccc}
\hline Time period & $\begin{array}{c}\text { Expansion } \\
\text { area(hm²) }\end{array}$ & $\begin{array}{c}\text { Expansion } \\
\text { percentage } \\
(\%)\end{array}$ & $\begin{array}{c}\text { Expansion } \\
\text { speed }\left(\mathrm{hm}^{2} / \mathrm{a}\right.\end{array}$ & $\begin{array}{c}\text { Expansi } \\
\text { on } \\
\text { rate }(\%)\end{array}$ & $\begin{array}{c}\text { Extended } \\
\text { Strength } \\
\text { Index }\end{array}$ & Overall expansion trend \\
\hline 1987 to 1995 & 804.11 & 8.74 & 100.51 & 1.09 & 0.27 & Low speed expansion \\
1995 to 2000 & 987.36 & 9.87 & 197.47 & 1.97 & 0.54 & Accelerating expansion \\
2000 to 2006 & 1236.78 & 11.25 & 206.13 & 1.87 & 0.56 & Rapid expansion \\
2006 to 2011 & 2272.31 & 18.58 & 454.46 & 3.72 & 1.24 & High speed expansion \\
1987 to 2011 & 5300.56 & 57.59 & 220.86 & 2.40 & 0.60 & Rapid expansion \\
\hline
\end{tabular}

from 1987 to 1995 , the urban land area increased by $804.11 \mathrm{hm}^{2}$, the expansion percentage was $8.74 \%$, the average annual expansion area was $100.51 \mathrm{hm}^{2}$, and the expansion rate and expansion intensity index were respectively $1.09 \%$ and 0.27 . The urban expansion intensity was relatively low, the cities and towns were at low expansion speed stage. From 1995 to 2000, the urban land area increased by $987.36 \mathrm{hm}^{2}$, the expansion percentage was $9.87 \%$, the expansion speed was $197.47 \mathrm{hm}^{2} / \mathrm{a}$, the expansion rate and expansion intensity index were $1.97 \%$ and 0.54 respectively, the urban expansion intensity increased significantly, and the cities and towns were in an accelerated expansion stage. From 2000 to 2006, the expansion area of urban land was still increasing, but the expansion rate slowed down compared with the previous period. The reason was that the rate of the rural residential land use had slowed down, and the cities and towns were in a rapid expansion stage. From 2006 to 2011, the rapid expansion of the central urban land area of Ganzhou district led to a rapid increase of the total land area, with an expansion rate of $454.46 \mathrm{hm}^{2} / \mathrm{a}$ and an expansion intensity index of 1.24, and the cities and towns entered a new phase of high-speed expansion (Figure 1).

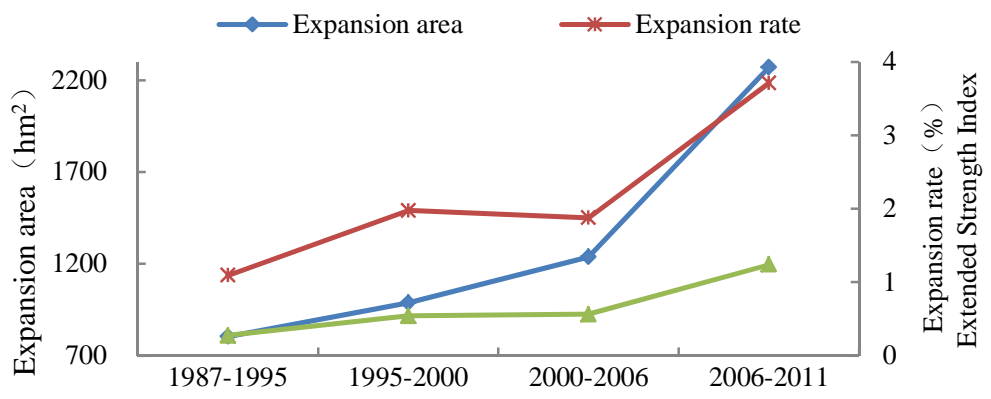

Fig.1 Changes of expansion area, speed and strength in Ganzhou County

4.2 Spatial Characteristics of Urban Expansion Fig. 2 shows the urban land area of Ganzhou District was expanded in different fan-shaped areas at different times. From 1987 to 2011, the urban land of Ganzhou District expanded in all directions, with the largest expansion area was in the Northwest, which was $1109.77 \mathrm{hm}^{2}$, and the extended azimuthal index was 20.94. In the northeast, the expansion area was $743.17 \mathrm{hm}^{2}$, the extended azimuthal index was 14.02 ; the expansion area in the southwest was $476.64 \mathrm{hm}^{2}$, which was the least, and the extended azimuthal index is 8.99 . The standard deviation of the town's extended azimuth index was 3.69, and the degree of data dispersion was low. Except for high-speed extension in the Northwestern and low-speed extension in the Southwest, the other directions were medium-speed expansion, and the difference in the expansion intensity was not significant. 


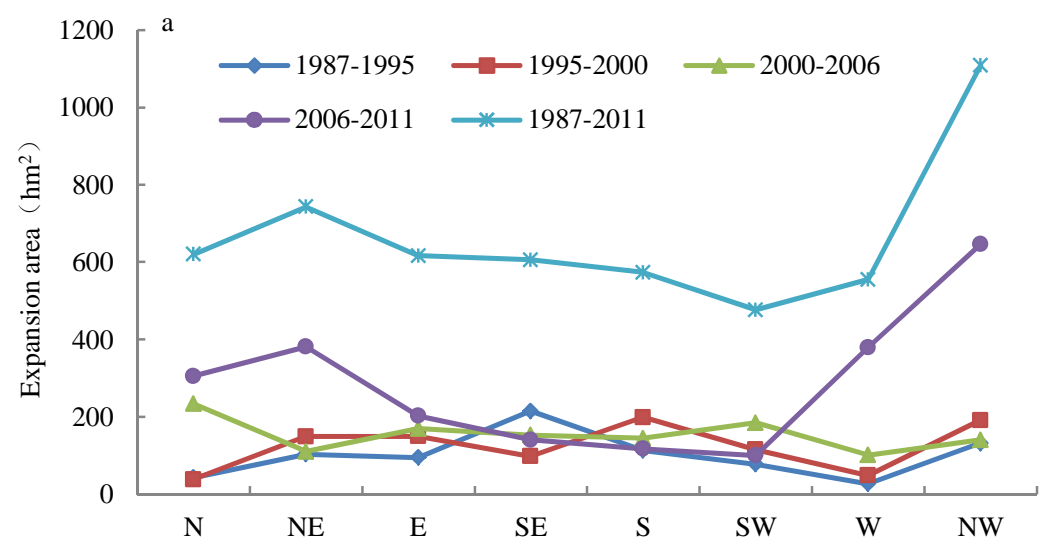

Fig. 2 The urban expansion area in all directions of Ganzhou County from 1987 to 2011

Formula (3) is used to calculate the extended orientation index for each azimuth in different periods, which showed in Tab.2. From 1987 to 1995, the standard deviation of the extended orientation index of urban space is 7.21 , and the data dispersion degree was relatively high, which indicated that there were significant differences in the expansion strength of urban space in each direction during this period. The Southeast was the main expansion position, which extended by $214.57 \mathrm{hm}^{2}$. The expansion type in the Northwest was rapid expansion, while in the Northeast, East and Southwest were medium-speed expansions, the North and Southwest were low-speed expansions, and the West is basically stable, expansion index was very small, the extended orientation index was only 3.31. The overall expansion of urban space was slow. The southeast and northwest are the main extension wings, which related to the development of Zhangye Airport and the development of townships such as Shajing and Wujiang along the Lanzhou-Xinjiang Railway.

Tab.2 Statistics of index of urban expansion azimuth in different periods

\begin{tabular}{cccccccccc}
\hline Time period & N & NE & E & SE & S & SW & W & NW & Standard deviation \\
\hline 1987 to 1995 & 5.33 & 12.81 & 11.78 & 26.68 & 14.06 & 9.64 & 3.31 & 16.39 & 7.21 \\
1995 to 2000 & 3.88 & 15.09 & 15.13 & 9.91 & 20.12 & 11.64 & 4.89 & 19.34 & 6.07 \\
2000 to 2006 & 18.90 & 8.90 & 13.71 & 12.36 & 11.69 & 14.90 & 8.17 & 11.37 & 3.42 \\
2006 to 2011 & 13.44 & 16.77 & 8.91 & 6.18 & 5.16 & 4.39 & 16.69 & 28.45 & 8.14 \\
1987 to 2011 & 11.70 & 14.02 & 11.62 & 11.43 & 10.82 & 8.99 & 10.47 & 20.94 & 3.69 \\
\hline
\end{tabular}

From 1995 to 2000, there were four main expansion directions: North, Northwest, East and Northeast. The expanded areas were $198.63 \mathrm{hm}^{2}, 190.98 \mathrm{hm}^{2}, 149.34 \mathrm{hm}^{2}$, and $149.01 \mathrm{hm}^{2}$, respectively, and the extended orientation index was greater than 15 , so the expansion type in the South was high-speed expansion type; followed by Southwest and Southeast positions, with an expanded area of $114.96 \mathrm{hm}^{2}$ and $97.85 \mathrm{hm}^{2}$, which were medium expansion types; the expansion areas in the West and North were the smallest, and extended orientation indexes do not exceed 5, and are basically stable types. The standard deviation of the azimuth index of urban extended orientation index is 6.07, and the degree of dispersion has decreased compared with the previous period, indicating that the difference in the expansion strength of urban space in each azimuth is reduced.

From 2000 to 2006, the standard deviation of the extended orientation index of urban space was 3.42 , and fluctuation range of the data was relatively small, indicating that the difference in the expansion strength of the urban space in each direction was the smallest. There were no stable and high-speed expansion orientation during this period, and all types of expansion were mainly medium-speed extended types. In the North, due to the construction of the Tuobaba Industrial Park, the area of expansion was the largest $\left(233.74 \mathrm{hm}^{2}\right)$, the extended orientation index is 18.90 , which was the rapid expansion type; the Southwest position is due to the development of townships such as Xiaoman and Longqu, and the construction of a high-capacity industrial park., the expansion of the 
downtown area to the West had a large area of expansion, the extended orientation index was 14.90, and expansion area in the East, Southeast, South, and Northwest were relatively big, which occupied more than $50 \%$ of the total expansion areas, the expansion types were medium expansion; the West and Northeast expansions areas were the smallest, and were the low speed expansion.

From 2006 to 2011, the spatial expansion direction of urban space in Ganzhou District was significantly affected by the expansion of the central urban land use. The expansion of Northwest azimuth was the most obvious, with an expansion of $646.36 \mathrm{hm}^{2}$ and an extended strength index was 28.45, which was a high speed expansion type; the expanded areas in Northeast and West were $381.10 \mathrm{hm}^{2}$ and $379.28 \mathrm{hm}^{2}$, respectively, and the extended orientation index were 16.77 and 16.69 , respectively, which were rapid expansion types. Among the other positions, the expansion type in the East was medium-speed expansion, and in the East, Southeast, and South, the expansion type were Low speed expansion models. The extended orientation index in Southwestern was only 4.39 and was stable. The standard deviation of the extended orientation index of urban is 8.14, and the data dispersion degree was the highest, which indicated that the difference in the expansion strength of the urban space in each direction were the most obvious.

Figure 3 is the plotted radar map, which was used to intuitively reflect the urban expansion space.
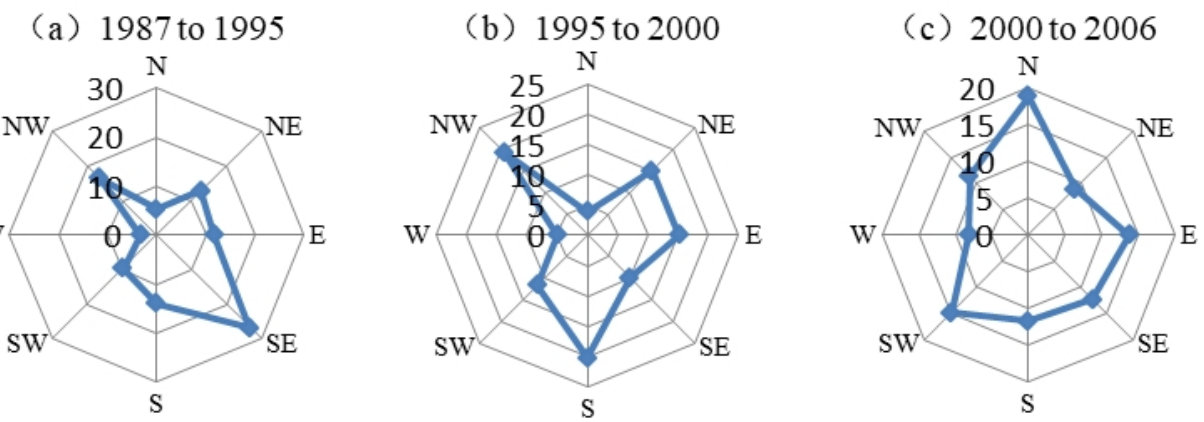

(d) 2006 to 2011

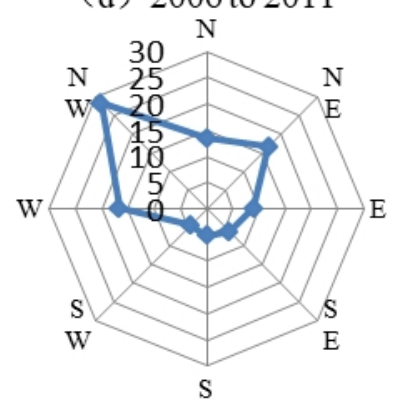

(e) 1987 to 2011

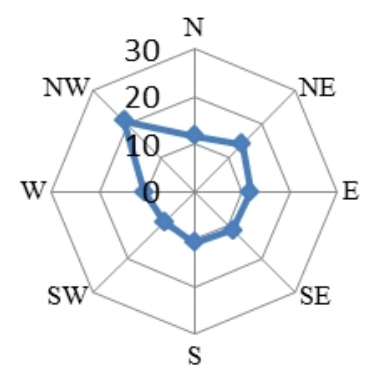

Fig. 3 Expansion orientation of different period in Ganzhou County

\section{Conclusion}

This paper mainly analyzes the spatial-temporal process and characteristics of cities and towns in Ganzhou District, and quantitatively analyzes the time course and spatial differentiation of urban expansion in Ganzhou District by using the urban expansion model and equal fan analysis method. The main conclusions are as follows:

(1) From 1987 to 2011, the urban land area in Ganzhou District increased 5305.56hm², which increased by $57.59 \%$, with an average annual increase of $220.86 \mathrm{hm}^{2}$. The expansion rate was $2.40 \%$, the expansion intensity index was 0.60 , and the urban land use expanded rapidly. Affected by the expansion of the central city and the construction of some industrial parks, the towns of Ganzhou District mainly expand to the Northwest, Northeast, and East.

(2) Judging from the time period, from 1987 to 1995, the urban land area increased by $804.11 \mathrm{hm}^{2}$, and was in a low speed expansion stage. Affected by the airport construction and traffic advantages, the urban land areas mainly expanded to the Southeast and Northwest, and the differences in the 
expansion strength of all positions were significant. From 1995 to 2000, the urban land area increased by $987.36 \mathrm{hm}^{2}$, and the towns entered an accelerated expansion phase; the towns mainly expanded to the South, Northeast, Northwest and the East. From 2000 to 2006, the urban land area increased by $1,236.78 \mathrm{hm}^{2}$, and the towns were in a rapid expansion stage; due to the construction of the Tuerba Industrial Park, the urban land mainly expanded to the North, followed by the Southwest and the East. From 2006 to 2011, the urban land area increased by $2272.31 \mathrm{hm}^{2}$, and the towns entered in a phase of rapid expansion; and the urban expansion mainly expanded to the Northwest, followed by the Northeast and West.

(3) The expansion of oasis cities and towns was constrained by natural resources such as water and land resources, as well as social and economic factors, transportation factors, land occupancy costs, and policy planning. This paper summarizes the spatial-temporal evolution characteristics of urban expansion in Oasis, how to clarify the influencing factors of the spatial-temporal evolution of urban expansion in Oasis, and predicts the scale and expansion direction of urban expansion in Oasis.

\section{Acknowledgements}

This work was financially supported by the National Natural Science Foundation of China (41661035) and Young Teachers Scientific Research Ability Promotion Plan of Northwest Normal University (NWNU-LKQN-14-14).

\section{References}

[1] Qiao W F,Sheng Y H,Fang B,et al. Land use change information mining in highly urbanized area based on transfer matrix:A case study of Suzhou,Jiangsu Province. Geographical Research,2013,08:1497-1507.

[2] Qin P,Guo D M. A study on the Expansion of the Urban Land Use in Qingdao. China Population, Resources and Environment,2016,26 (5):401-404.

[3] Liu S H,Wu C J,Shen H Q. A GIS based Model of Urban Land Use Growth in Beijing. Acta Geographica Sinica,2000,55(4):407-416.

[4] Qiang H Y,Zhang X L,Lei J. Oasis urban system in arid area of Xinjiang based on fractal theory. Arid Land Geography,2010,33(5):802-808.

[5] Ma G X,Gan G H,Tian Y J. Development Models of Zhangye Oasis Cities and Towns under Restraint of Water Resources. Journal of Desert Research,2006,26(3):426-431.

[6] Hassan M M,Nazem M N I. Examination of Land Use /land Cover Changes,Urban Growth Dynamics,and Environmental Sustainability in Chittagong City,Bangladesh. Environment,Development and Sustainability,2016,18:697-716.

[7] Ma R H,Gu C L,Pu Y X,et al. Urban Spatial Sprawl Pattern and Metrics in South of Jiangsu Province along the Yangtze River. Acta Geographica Sinica,2007,62(10):1011-1022.

[8] Ma X K,Gao M H. Dynamic assessment of land ecologic safety of oasis city in arid northwest China:a case of Korla City in Xinjiang. Arid Land Geography,2017,40(1):172-180. 